\title{
Relationship between Changes in Fatigue and Exercise by Follow-Up Period
}

\author{
Seung Min Oh', Woo Kyung Bae ${ }^{2, *}$, Se Ryung Choo ${ }^{3}$, Hee Tae Kim', Hyun Ho Kim', Sang Hyun Lee', Han Sol Jeong' \\ ${ }^{1}$ Department of Family Medicine, Seoul National University College of Medicine, Seoul, Korea \\ ${ }^{2}$ Health Promotion Center, Seoul National University Bundang Hospital, Seongnam, Korea \\ ${ }^{3}$ Department of Family Medicine, Seoul National University Bundang Hospital, Seongnam, Korea
}

Background: Fatigue is one of the most common presenting symptoms in primary care in Korea. In this study, we aimed to determine the effect of exercise intervention on the severity of fatigue of unknown medical cause during a period of follow-up.

Methods: We used the data collected from an outpatient fatigue clinic in Seoul National University Bundang Hospital. The study was conducted from March 3, 2010 to May 31, 2014. We measured the body mass index of each patient and evaluated variables including lifestyle factors (smoking, alcohol consumption, and regular exercise), quality of sleep, anxiety, depression, stress severity, and fatigue severity using questionnaires. A total of 152 participants who completed questionnaires to determine changes in fatigue severity and the effect of exercise for each period were evaluated. We used univariate analysis to verify possible factors related to fatigue and then conducted multivariate analysis using these factors and the literature.

Results: Of 130 patients with the complaint of chronic fatigue for over 6 months, over 90 percent reported moderate or severe fatigue on the Fatigue Severity Scale and Brief Fatigue Inventory questionnaires. The fatigue severity scores decreased and fatigue improved over time. The amount of exercise was increased in the first month, but decreased afterwards.

Conclusion: There was no significant relationship between changes in the amount of exercise and fatigue severity in each follow-up period. Randomized controlled trials and a cohort study with a more detailed exercise protocol in an outpatient setting are needed in the future.

Keywords: Fatigue; Physical Activity; Life Style; Metabolic Equivalent 


\section{INTRODUCTION}

Fatigue is a symptom that is commonly seen in primary care. A study of 1,648 patients over 18 years of age in eight local primary care institutions reported that $21.1 \%$ complained of persistent fatigue for six months or less, and $8.4 \%$ reported chronic fatigue for more than 6 months. ${ }^{1)}$ Chronic fatigue prevention and treatment is necessary because it interferes with activities of daily living and exerts a major impact on quality of life. ${ }^{2)}$ However, there is no standardized questionnaire for fatigue severity since the level of perceived fatigue is different for each person, and the physical, mental, and socioeconomic causes are diverse. ${ }^{3)}$ For these reasons, fatigue is difficult to study.

The causes of chronic fatigue in the primary health care study in Korea 2005 were mental factors, around $46 \%$ (stress, depression, adjustment disorders, anxiety disorders, sleep disorders, hwa-byung, alcoholism, and panic disorder), organic factors, approximately $34 \%$ (uncontrolled diabetes, asthma, chronic hepatitis, tuberculosis, thyroid function abnormality, iron deficiency anemia, fatty liver disease, sleep apnea, chronic obstructive pulmonary disease, and herniated intervertebral disc), and unknown factors, about 20\%. ${ }^{12}$ It has been reported that factors related to fatigue include age, gender, body mass index (BMI), lifestyle (smoking, alcohol consumption, regular exercise, and caffeine intake), psychological state (stress, depression, and anxiety), marital status, social life, occupation, sleep disorder, and autonomic nervous system. ${ }^{1,4,5)}$

Most previous studies considered specific conditions known to be associated with fatigue, such as illnesses (cancer, ${ }^{6}$ ischemic heart disease, ${ }^{7)}$ systemic lupus erythematosus, ${ }^{8)}$ multiple sclerosis, ${ }^{9)}$ etc.), and sleep disorders, ${ }^{10)}$ anxiety, or depression. ${ }^{11)}$ However, there are few studies of chronic fatigue without known organic cause in the primary care setting. In a study with a primary care setting, negative life events and lack of physical fitness were related with new onset fatigue. ${ }^{12)}$ In another study, chronic fatigue symptoms treated with counseling and graded exercise for one year improved, compared to a usual care control group, but the improvement was not significant. ${ }^{13)}$ There have been randomized controlled trials on the effects of aquatic exercise in women who complained of joint pain, ${ }^{14)}$ and the effect of walking exercise intensity on fatigue and blood lipid changes in middle-aged women, ${ }^{15)}$ but these studies were limited by being conducted on a small number of specific conditions, and did not consider previous known factors that can cause fatigue.

The present study is limited to fatigue patients without an organic cause, such as malignancy, uncontrolled diabetes, asthma, chronic hepatitis, tuberculosis, ischemic heart disease, thyroid dysfunction, or iron deficiency anemia. Lifestyle and physical factors that exacerbate fatigue, such as obesity, smoking, drinking, and sleep disorders, and intermittent medication for mental factors related to fatigue, such as anxiety, depression, stress, and sleep disorders, were evaluated in this study. We assessed the effect of changes in the amount of exercise after initiating treatment interventions on changes in the severity of fatigue according to follow-up periods over time.

\section{METHODS}

\section{Study Population}

This study was conducted on patients who visited a fatigue clinic in the department of family medicine, Seoul National University Bundang Hospital, from March 3, 2010 to May 31, 2014. We excluded 145 patients who only visited once, 38 who did not complete the exercise questionnaire after the first visit, and 4 who did not return to the clinic within 6 months. Finally, we analyzed 152 patients for whom exercise and fatigue changes could be determined for each follow-up period.

\section{Study Procedures}

We recorded gender, age, and BMI at the first visit, and smoking, drinking, exercise, fatigue, sleep quality, anxiety, depression, and stress level were investigated through a self-reporting survey. Follow-up during the study period was performed at 3 time points: within 1 month after the first visit, within 1 to 3 months, and within 3 to 6 months.

\section{1) Age, body mass index, social behaviors}

Age and BMI were classified as continuous variables, and smoking was counted as a categorical variable, reported as 'never smoked,' 'exsmoker', or 'current smoker'. Drinking was categorized as 'none or average drinker' or 'current drinker.' Risk drinking was defined as over 40 $\mathrm{g}$ of alcohol daily for men and $20 \mathrm{~g}$ for women using the World Health Organization criteria. ${ }^{16)}$

\section{2) Fatigue}

Evaluation of fatigue was conducted using the Fatigue Severity Scale (FSS) and the Brief Fatigue Inventory (BFI). The FSS is widely used to examine the effects of fatigue, and has high reproducibility among the most common fatigue surveys. ${ }^{3)}$ The validity of the Korean version of FSS questionnaire was demonstrated. ${ }^{17)}$ FSS assesses the status of fatigue for the prior week using 9 questions graded from 1 to 7 points for each item, and the average is calculated. A higher score means a greater degree of fatigue. We classified the high fatigue group as having a score of more than 4.5 points, moderate as 3 to 4.5 points, and mild as less than 3 points, with reference to the literature on FSS. ${ }^{3,17}$

The BFI was developed to rapidly measure the degree of fatigue in cancer patients, ${ }^{18)}$ and the reliability and validity of the Korean version was has been reported. ${ }^{19)}$ The usefulness of the BFI for evaluating fatigue severity and associated factors in the general population has been documented in community research. ${ }^{20)}$ The BFI consists of 9 questions on fatigue and its interference with daily life in the prior 24 hours, and is scored from 0 to 10 points for each question, plus the total. An increase in the average score means that the severity of fatigue is increased. The first 3 questions ask the subject to describe the current level of fatigue, the usual level of fatigue during the previous 24 hours, and the maximum level of fatigue during the previous 24 hours. Unlike the next 6 questions about the relationship between the average level of functioning and mood, the first 3 questions measure the severity of fatigue, and we used these to calculate the change in level of 
fatigue in this study. ${ }^{20)}$ We classified the severe fatigue group as those who scored at least 7 points by reference to the literature, moderate as 4 to less than 7 points, and mild as less than 4 points. ${ }^{19)}$

\section{3) Exercise amount}

The type of exercise reported in medical records and questionnaires was converted into dynamic physical activity according to metabolic equivalents (METs). We converted values to minutes of running time for workout activities performed continuously for at least 10 minutes at a time in one week, and then summed the values to classify a 'low exercise group (600 MET-min/wk or less),' a 'moderate exercise group (600 to 1,500 MET-min/wk or less),' and a 'high exercise group (1,500 MET-min/wk or more)., ${ }^{21)}$

\section{4) Sleep quality}

Quality of sleep was evaluated by the Pittsburgh Sleep Quality Index (PSQI), which was developed to rate the quality of sleep and identify sleep disorders. The PSQI assesses subjective sleep quality, latency, duration, habitual sleep behavior, sleep disturbance, sleep drug use, and daytime dysfunction. Each item is scored as 0 to 3 points; we classified 'good sleep quality' as a score of 5 points or less and 'bad sleep quality' as 6 points or more. ${ }^{22)}$

\section{5) Anxiety and depression}

Anxiety and depression were evaluated using the Korean version of the short Goldberg Anxiety and Depression Scale. The Goldberg scale has been proven to identify anxiety and depression, and the Korean version has been validated. ${ }^{23)}$ It consists of 18 questions, including 4 for major symptoms and 5 for minor symptoms for each of anxiety and depression. Each item is scored as 0 or 1 point, and the higher the total score, the more severe the anxiety and depression. We classified the 'anxious' group by a score of 5 or more points for anxiety-related questions and the 'depressed' group by a score of 5 or more points for depression-related questions. ${ }^{24)}$

\section{6) Stress}

The amount of stress was measured using the Korean version of the Brief Encounter Psychosocial Instrument (BEPSI-K). The BEPSI-K comprises 5 questions scored on a 5-point scale; the greater the total, the higher the level of stress. We allocated the subjects with a score of 2.4 or more points in the "stressed group"25)

\section{Statistical Analysis}

Univariate analysis was performed, with correlation analysis, t-tests, and analysis of variance comparing fatigue scores in each follow-up period and variables such as gender, age, BMI, smoking, alcohol, anxiety, depression, stress, and the quality of sleep. Multiple linear regression analysis compared changes in level of fatigue and the change in the amount of exercise in each follow-up period, including fatigue-associated disturbance variables. Statistical significance was set at a level of less than 5\%. We used STATA SE ver. 12.1 (Stata Co., College Station,
TX, USA) for data analysis.

\section{RESULTS}

\section{Baseline Characteristics}

Of 152 subjects, there were more females (87) than males (65), and the mean age was 51.9 years. There were 130 non-smokers and ex-smokers (86.6\%), and 74 none or average drinkers (48.7\%). Those who complained of chronic fatigue for 6 months or more accounted for 130 patients (85.5\%). There were 138 with moderate to severe fatigue on the FSS (91.4\%), and 135 (88.8\%), 140 (92.1\%), and 146 (96.0\%) on the first 3 questions of the BFI, respectively. In the initial survey, there were 89 (60.1\%) who performed moderate or greater physical activity. There were 96 (76.8\%) who complained of poor sleep quality, and 87 (60.0\%) with suspected anxiety disorder. There were 96 patients who visited 3 times or less, and 13 who visited more than 5 times (Table 1 ).

\section{Subject Numbers, Exercise Amount, and Fatigue Scale Differences in Each Follow-Up Period}

Follow-up time points were categorized as within 1 month, 1 to 3 months, and 3 to 6 months after the first fatigue clinic visit, respectively. A total of 152 cases were studied after all fatigue and exercise surveys were completed, and then the data for the 109, 64, and 39 subjects tracked during the above respective time periods were analyzed. All levels of fatigue decreased with time, but the amount of exercise only increased within the first month, and decreased thereafter (Table 2).

\section{Correlation between Exercise Amount and Fatigue in Each Follow-Up Period}

We conducted correlation analysis comparing changes in level of fatigue using 4 questionnaire scores and changes in exercise amount to calculate results for initial and final visit survey data in each period. There was a negative association $(\mathrm{P}=0.02)$ between the amount of exercise change in the period from 3 to 6 months after the first visit and the change in level of fatigue described at the time of the visit; there was also a negative association between the change in the BMI and changes in the average fatigue level for the prior 24 hours $(\mathrm{P}=0.02)$. Women tended to show more improvement than men in the changes in level of fatigue for the prior day $(\mathrm{P}=0.03)$ and for the maximum fatigue level in the prior 24 hours $(\mathrm{P}=0.01)$ in the period of follow-up within one month. During the same period, fatigue improvement in current drinkers tended to be less than that in none or average drink$\operatorname{ers}(\mathrm{P}=0.01)$.

\section{Multivariate Analysis of Fatigue Changes according to Differences in Activity in Each Follow-Up Period}

Based on the univariate analysis and the literature, we performed multiple linear regression analysis for confounding variables associated with fatigue, such as anxiety, depression, stress, quality of sleep, fatigue duration, gender, age, BMI, smoking, and alcohol consumption. The change in fatigue level in each period was calculated using the 4 differ- 
Table 1. Baseline characteristics of the subjects $(n=152)$

\begin{tabular}{|c|c|}
\hline Characteristic & Value \\
\hline Male sex & $65(42.8)$ \\
\hline Age (v) & $51.9 \pm 12.1$ \\
\hline Body mass index $\left(\mathrm{kg} / \mathrm{m}^{2}\right)$ & $22.6 \pm 3.3$ \\
\hline \multicolumn{2}{|l|}{ Smoking } \\
\hline Non-smoker & 89 (59.3) \\
\hline Ex-smoker & $41(27.3)$ \\
\hline Current smoker & $20(13.3)$ \\
\hline \multicolumn{2}{|l|}{ Drinking } \\
\hline None or average drinker & $74(48.7)$ \\
\hline Current drinker & 78 (51.3) \\
\hline \multicolumn{2}{|l|}{ Fatigue duration* (mo) } \\
\hline$<1$ & $7(4.6)$ \\
\hline $1-6$ & $15(9.9)$ \\
\hline$\geq 6$ & $130(85.5)$ \\
\hline Clinic visit no. (times) & $3.9 \pm 0.2$ \\
\hline$<3$ & $96(63.2)$ \\
\hline $3-5$ & $43(28.3)$ \\
\hline$\geq 5$ & $13(8.5)$ \\
\hline Exercise amount (metabolic equivalents-min/wk) & $993.8 \pm 1661.6$ \\
\hline Low $(<600)$ & $59(39.9)$ \\
\hline Moderate $(600-1,500)$ & $62(41.9)$ \\
\hline High $(\geq 1,500)$ & 27 (18.2) \\
\hline Fatigue Severity Score-total average (points) & $5.0 \pm 1.4$ \\
\hline Mild $(<3)$ & $13(8.6)$ \\
\hline Moderate (3-4.5) & $34(22.5)$ \\
\hline High ( $\geq 4.5)$ & $104(68.9)$ \\
\hline Fatigue right now ${ }^{\dagger}$ (points) & $6.3 \pm 2.0$ \\
\hline Mild $(<4)$ & $17(11.2)$ \\
\hline Moderate (4-7) & $55(36.2)$ \\
\hline Severe $(\geq 7)$ & $80(52.6)$ \\
\hline Usual fatigue in previous 24 hours (points) & $6.6 \pm 2.0$ \\
\hline Mild $(<4)$ & $12(7.9)$ \\
\hline Moderate (4-7) & $57(37.5)$ \\
\hline Severe $(\geq 7)$ & $83(54.6)$ \\
\hline Worst fatigue in previous 24 hours $^{\S}$ (points) & $7.5 \pm 1.9$ \\
\hline Mild $(<4)$ & $6(4.0)$ \\
\hline Moderate (4-7) & $34(22.3)$ \\
\hline Severe $(\geq 7)$ & $112(73.7)$ \\
\hline Goldberg Depression Scale (points) & $4.1 \pm 2.1$ \\
\hline Non-depressed $(<5)$ & $82(56.2)$ \\
\hline Depressed $(\geq 5)$ & $64(43.8)$ \\
\hline Goldberg Anxiety Scale (points) & $5.1 \pm 2.3$ \\
\hline Non-anxious $(<5)$ & $58(40.0)$ \\
\hline Anxious $(\geq 5)$ & $87(60.0)$ \\
\hline Brief Encounter Psychosocial Instrument-Korea for stress (points) & $10.8 \pm 3.9$ \\
\hline Non-stressed $(<2.4)$ & $94(65.3)$ \\
\hline Stressed $(\geq 2.4)$ & $50(34.7)$ \\
\hline Pittsburgh Sleep Quality Index global score for sleep quality (points) & $8.7 \pm 3.8$ \\
\hline Good sleep quality (5) & $29(23.2)$ \\
\hline Poor sleep quality $(>5)$ & 96 (76.8) \\
\hline
\end{tabular}

Values are presented as number (\%) or mean \pm standard deviation.

Goldberg's, Goldberg's Short Screening Scale; BFI, Brief Fatigue Inventory.

*Fatigue duration is total months of fatigue when patients visit the clinic initially.

${ }^{\dagger}$ Question number 1 of $\mathrm{BFI}$ items describes fatigue severity level when asked.

${ }^{\ddagger}$ Question number 2 of $\mathrm{BFI}$ items describes fatigue at its usual level during previous

24 hours. ${ }^{\S}$ Question number 3 of BFl items describes fatigue at its worst level during previous 24 hours. ent questionnaire scores at the initial and final visit of each follow-up period. The FSS was not statistically significant in any follow-up period (P-value $=0.45,0.25,0.79$ at less than 1 month, 1 to 3 months, 3 to 6 months, respectively). The response to BFI question 1 regarding fatigue changes at the time of questionnaire completion was not statistically significant (P-value $=0.3,0.27,0.16$ at less than 1 month, 1 to 3 months, 3 to 6 months, respectively); the response to BFI question 2 about average fatigue changes during the prior 24 hours ( $\mathrm{P}$-value $=0.22$, 0.99, 0.53 at less than 1 month, 1 to 3 months, 3 to 6 months, respectively) also was not significant. The response to BFI question 3 describing fatigue during the prior 24 hours also showed no statistical significance (P-value $=0.94,0.49,0.47$ at less than 1 month, 1 to 3 months, 3 to 6 months, respectively). There was no statistically significant relationship between the change in the amount of exercise and the change in level of fatigue in each follow-up period after the initial visit in multiple linear regression analysis (Table 3 ).

\section{DISCUSSION}

The BFI used in this research has clinical usefulness in a survey measuring severe fatigue, and was also found to be suitable for measurement of chronic, moderate, and severe fatigue in another study. ${ }^{18)}$ The FSS was used with the BFI in this study because ths FSS can be used to discriminate fatigue from mental illness when used together with anxiety and depression measurement tools. ${ }^{17)}$ With the BFI and the FSS, we identified the levels of overall fatigue and fatigue within one day at each visits following the initial visit, and measured the changes in each fatigue scale.

This study targeted a specific group of patients whose fatigue was not caused by an organic disease or drug. We used questionnaires to assess anxiety, depression, stress, and quality of sleep to identify mental factors related to fatigue, so the study has greater implications for effective intervention and proper treatment in many primary care outpatient clinics managing patients with fatigue. ${ }^{2)}$

We divided the follow-up periods into 3 categories after the first clinic visit, and subdivided the changes for each variable such as exercise amount and fatigue, and for multiple covariables by each time category. The possibility that fatigue could worsen with additional exercise in an early phase was also considered.

Fatigue is a subjective representation, and the definition is somewhat group-dependent; ${ }^{4)}$ there are differences in the measurement of fatigue depending on the aspects of interest to the subjects being assessed. The FSS questionnaire specifically addresses whether subjects feel tired after exercise and whether they experience dysfunction after working out, but we did not use that tool efficiently, because it is limited to measurement of average final scores. The BFI is helpful in understanding the relevance of fatigue in both the general population and in patients, ${ }^{4)}$ but requires further research on intervention and treatment for improvement of function in moderate or mild fatigue. ${ }^{18)}$

In general, regular exercise is traditionally known to reduce fatigue in studies on both men and women. ${ }^{4)}$ In this study, the subjects were a 
Table 2. Subject numbers, exercise amount, and fatigue scale differences at each follow-up period

\begin{tabular}{|c|c|c|c|}
\hline Variable & Within 1 month $(n=109)$ & 1 to 3 months $(n=64)$ & 3 to 6 months $(n=39)$ \\
\hline Exercise amount (metabolic equivalents-min/wk) & $201.76 \pm 1,095.76$ & $-22.13 \pm 1,005.87$ & $-43.72 \pm 662.43$ \\
\hline Fatigue Severity Score average & $-0.43 \pm 1.07$ & $-0.30 \pm 0.86$ & $-0.05 \pm 1.31$ \\
\hline Fatigue right now ${ }^{\star}$ & $-0.59 \pm 2.35$ & $-0.41 \pm 1.78$ & $-1.00 \pm 2.40$ \\
\hline Usual fatigue in previous 24 hours $^{\dagger}$ & $-0.74 \pm 2.23$ & $-0.63 \pm 1.77$ & $-0.64 \pm 2.02$ \\
\hline Worst fatigue in previous 24 hours $^{\ddagger}$ & $-0.78 \pm 2.10$ & $-0.67 \pm 1.79$ & $-0.74 \pm 2.12$ \\
\hline
\end{tabular}

The number of patients who had their own data for fatigue and exercise changes in each period.

${ }^{*}$ Question number 1 of Brief Fatigue Inventory items describes fatigue right now. ${ }^{\dagger}$ Question number 2 of Brief Fatigue Inventory items describes fatigue at its usual level in previous 24 hours. ${ }^{\ddagger}$ Question number 3 of Brief Fatigue Inventory items describes fatigue at its worst level in previous 24 hours.

Table 3. Multivariant analysis for fatigue changes with activity differences in each follow-up period

\begin{tabular}{|c|c|c|c|}
\hline Fatigue scale in specific period (mo) & Coefficient* & $\begin{array}{l}\text { 95\% confidence } \\
\text { interval }\end{array}$ & P-value \\
\hline \multicolumn{4}{|l|}{ Fatigue Severity Score average score } \\
\hline$<1$ & 0.07 & -0.12 to 0.26 & 0.45 \\
\hline $1-3$ & 0.11 & -0.08 to 0.31 & 0.25 \\
\hline $3-6$ & -0.14 & -1.23 to 0.95 & 0.79 \\
\hline \multicolumn{4}{|l|}{ Fatigue right now ${ }^{\dagger}$} \\
\hline$<1$ & -0.23 & -0.68 to 0.21 & 0.3 \\
\hline $1-3$ & 0.25 & -0.2 to 0.7 & 0.27 \\
\hline $3-6$ & -1.45 & -3.52 to 0.62 & 0.16 \\
\hline \multicolumn{4}{|l|}{ Usual fatigue in previous 24 hours $^{\ddagger}$} \\
\hline$<1$ & -0.26 & -0.68 to 0.16 & 0.22 \\
\hline $1-3$ & -0.004 & -0.5 to 0.49 & 0.99 \\
\hline $3-6$ & -0.51 & -2.2 to 1.17 & 0.53 \\
\hline \multicolumn{4}{|l|}{ Worst fatigue in previous 24 hours ${ }^{\S}$} \\
\hline$<1$ & -0.02 & -0.39 to 0.36 & 0.94 \\
\hline $1-3$ & 0.17 & -0.31 to 0.65 & 0.49 \\
\hline $3-6$ & -0.66 & -2.53 to 1.2 & 0.47 \\
\hline
\end{tabular}

*Linear regression method was used for analysis between comparing scale changes and activity amount (1,000 metabolic equivalents-min/wk) changes with several covariate variables; sex, age, body mass index, smoking, drinking, anxiety, depression, stress, and sleep quality at initial visit. ${ }^{\dagger}$ Question number 1 of Brief Fatigue Inventory items describes fatigue right now at its usual level. ₹Question number 2 of Brief Fatigue Inventory items describes fatigue at its usual level during previous 24 hours. ${ }^{\S}$ Question number 3 of Brief Fatigue Inventory items describes fatigue at its worst level during previous 24 hours.

specific group of patients who visited the fatigue clinic, but had no underlying disease. Among the subjects, $60.5 \%$ were already generally physically active or worked out with specific exercises at moderate intensity at the time of the initial visit; therefore, they could have difficulty subjectively sensing the effect of exercise intervention on their level of fatigue. If the patients already performed exercise at the beginning of the study, they rarely changed the type, frequency, and intensity of exercise in the short term. We suggested that they perform their usual exercises as much as possible, so they were unlikely to perform additional prescribed exercise.

Improper or excessive exercise can worsen symptoms in chronic fatigue patients. Follow-up for graded exercise therapy (GET) for chronic fatigue syndrome ${ }^{13,26)}$ is limited to the primary setting since it requires regular exercise assessment, an exercise prescription, and payments for care. Subjects in this study tended to overwork, which primarily ac- counted for their chronic fatigue without an underlying organic basis; therefore, we initially recommended adequate rest instead of exercise. We added GET after fatigue improved, so it was difficult to determine whether the initial visit represented the initiation of exercise therapy. This observational study had limitations. The changes in amount of exercise were measured solely according to time, and the amount of exercise was increased very slowly to prevent fatigue exacerbation. Moreover, no constructive exercise education was provided.

The response rate for this exercise-related survey was low, and the duration and frequency of exercise were often limited, especially for time-consuming and high-cost activities such as climbing and outdoor golf. Regular exercise requires mental and physical preparation and more time and space. We documented recommendations for additional physical activity in the electronic medical records, but most of these lacked responses at the subsequent visit. Seasonal change, family events, falls, and traffic accidents were also major obstacles preventing patients from performing physical activity.

Some patients were on sedatives or antidepressants at the initial visit, and others sometimes started medication during the follow-up period for symptom relief. They tended to take medication intermittently, further limiting the validity of the results of this study.

Further high quality research is needed on the effect of changes in the amount of exercise and changes in the level of fatigue; a randomized, controlled study comparing exercise therapy with no intervention, or a prospective study emphasizing GET and follow-up of an outpatient clinic-based protocol, are needed. Detailed information about questionnaire completion should be given to patients beforehand, to obtain more accurate responses in a self-reported survey, and increased use of short-form questionnaires can increase the response rate. ${ }^{3)}$ The development of new tools for recording, such as smartphone applications or use of wearable devices, are also required to supplement deficiencies in survey responses.

Development of a program with a variety of exercises as interventional treatment is also important. Those working longer than 9 hours a day are known to be at higher risk of fatigue than the unemployed, ${ }^{2)}$ suggesting that time is needed to recover from the fatigue caused by overwork. Regular aerobic exercise is known to be effective for the quality of sleep in healthy older people with complaints of moderate sleep disorders. ${ }^{27)}$ Targeted 10-minute exercise in pilots deprived of sleep for 40 hours temporarily reduces fatigue because of increased 
alertness, but the fatigue eventually recurs within one hour after exercise ${ }^{28)}$ It is important to exercise regularly in sufficient quantities in a fatigue-free state in order to obtain the benefits of exercise. This means that motivation to exercise is needed for 'too busy and have no time to exercise' people, and those who are 'always tired.' Development of a simple exercise regimen that anyone can use without time and space limitations, implementation of interval training and lifestyle changes to increase activity, and raising awareness of the negative effects of fatigue to overcome them are also needed. Environmental constraints on individual physical activity can be greater than the individual's motivation to exercise, and promotion of physical activity and exercise is needed to enable social behaviors and actions that individuals can regulate. ${ }^{29)}$ Various types of exercise programs should be developed to increase motivation and compliance with physical activity; as one example, 'Wii Fit' is known to be effective in reducing fatigue in certain illnesses. ${ }^{30)}$

This study did not show a significant relationship between changes in fatigue and the amount of exercise according to the follow-up period after the initial visit to a clinic.

\section{CONFLICT OF INTEREST}

No potential conflict of interest relevant to this article was reported.

\section{REFERENCES}

1. Kim CH, Shin HC, Won CW. Prevalence of chronic fatigue and chronic fatigue syndrome in Korea: community-based primary care study. J Korean Med Sci 2005;20:529-34.

2. Song JI, Ahn EM, Kim KW, Gwak HK, Park MS, Yoo SH. Factors associated with self-reported fatigue: from Korean National Health and Nutrition Examination Survey 2001. J Korean Acad Fam Med 2007;28:83544.

3. Dittner AJ, Wessely SC, Brown RG. The assessment of fatigue: a practical guide for clinicians and researchers. J Psychosom Res 2004;56:15770.

4. Chang SJ, Koh SB, Kang MG, Hyun SJ, Cha BS, Park JK, et al. Correlates of self-rated fatigue in Korean employees. J Prev Med Public Health 2005;38:71-81.

5. Kim JM, Shin MJ, Lee SH, Choi WS, Ock SM, Kim CM, et al. The relationship between autonomous function and fatigue rating in patients with fatigue. J Korean Acad Fam Med 2004;25:52-8.

6. Stasi R, Abriani L, Beccaglia P, Terzoli E, Amadori S. Cancer-related fatigue: evolving concepts in evaluation and treatment. Cancer 2003;98: 1786-801.

7. Sandor B, Nagy A, Toth A, Rabai M, Mezey B, Csatho A, et al. Effects of moderate aerobic exercise training on hemorheological and laboratory parameters in ischemic heart disease patients. PLoS One 2014;9:e110751.

8. Ahn GE, Ramsey-Goldman R. Fatigue in systemic lupus erythematosus. Int J Clin Rheumtol 2012;7:217-27.

9. Veauthier C, Radbruch H, Gaede G, Pfueller CF, Dorr J, BellmannStrobl J, et al. Fatigue in multiple sclerosis is closely related to sleep disorders: a polysomnographic cross-sectional study. Mult Scler 2011; 17:613-22.

10. Lavidor M, Weller A, Babkoff H. How sleep is related to fatigue. Br J Health Psychol 2003;8(Pt 1):95-105.

11. McKercher C, Patton GC, Schmidt MD, Venn AJ, Dwyer T, Sanderson K. Physical activity and depression symptom profiles in young men and women with major depression. Psychosom Med 2013;75:366-74.

12. Chalder T, Neeleman J, Reme SE, Power M, Wessely S. Factors associated with acute fatigue in primary care. Psychol Med 2010;40:1289-95.

13. Ridsdale L, Hurley M, King M, McCrone P, Donaldson N. The effect of counselling, graded exercise and usual care for people with chronic fatigue in primary care: a randomized trial. Psychol Med 2012;42:221724.

14. Chang KO. The effects of an aquatic exercise program on pain, fatigue, physical fitness, disability in ADL, and psychological variables in women with arthritis. J Korean Acad Community Health Nurs 2009;20: 87-95.

15. Lee JI. Effects of walking exercise intensities on fatigue, serum lipids and immune function among middle-aged women. J Korean Acad Nurs 2006;36:94-102.

16. World Health Organization. Global strategy to reduce the harmful use of alcohol. Geneva: World Health Organization; 2010.

17. Chung KI, Song CH. Clinical usefulness of fatigue severity scale for patients with fatigue, and anxiety or depression. J Korean Psychosom Med 2001;9:164-73.

18. Mendoza TR, Wang XS, Cleeland CS, Morrissey M, Johnson BA, Wendt JK, et al. The rapid assessment of fatigue severity in cancer patients: use of the Brief Fatigue Inventory. Cancer 1999;85:1186-96.

19. Yun YH, Wang XS, Lee JS, Roh JW, Lee CG, Lee WS, et al. Validation study of the Korean version of the brief fatigue inventory. J Pain Symptom Manage 2005;29:165-72.

20. Yun YH, Lee MK, Chun HN, Lee YM, Park SM, Mendoza TR, et al. Fatigue in the general Korean population: application and normative data of the Brief Fatigue Inventory. J Pain Symptom Manage 2008;36: 259-67.

21. Ainsworth BE, Haskell WL, Herrmann SD, Meckes N, Bassett DR Jr, Tudor-Locke C, et al. 2011 Compendium of Physical Activities: a second update of codes and MET values. Med Sci Sports Exerc 2011;43: 1575-81.

22. Buysse DJ, Reynolds CF 3rd, Monk TH, Berman SR, Kupfer DJ. The Pittsburgh Sleep Quality Index: a new instrument for psychiatric practice and research. Psychiatry Res 1989;28:193-213.

23. Backhaus J, Junghanns K, Broocks A, Riemann D, Hohagen F. Test-retest reliability and validity of the Pittsburgh Sleep Quality Index in primary insomnia. J Psychosom Res 2002;53:737-40.

24. Kim JS, Kim YS, Lee GY, Park TJ, Lee YH, Kong BK, et al. The standardization of Korean-translated Goldberg's shart screening scale for anxiety and depression. J Korean Acad Fam Med 1997;18:1452-60.

25. Kim KN, Park JY, Shin TS, Jun KJ, Choi EY, Kim HJ, et al. Degree of stress and stress-related factors by the Korean version of the BEPSI. J Korean Acad Fam Med 1998;19:559-70.

26. Yancey JR, Thomas SM. Chronic fatigue syndrome: diagnosis and treatment. Am Fam Physician 2012;86:741-6.

27. King AC, Oman RF, Brassington GS, Bliwise DL, Haskell WL. Moderate-intensity exercise and self-rated quality of sleep in older adults: a 
randomized controlled trial. JAMA 1997;277:32-7.

28. JrLeDuc PA, Caldwell JA Jr, Ruyak PS. The effects of exercise as a countermeasure for fatigue in sleep-deprived aviators. Mil Psychol 2000;12:249-66.

29. Dishman RK, Sallis JF, Orenstein DR. The determinants of physical ac- tivity and exercise. Public Health Rep 1985;100:158-71.

30. Yuen HK, Holthaus K, Kamen DL, Sword DO, Breland HL. Using Wii Fit to reduce fatigue among African American women with systemic lupus erythematosus: a pilot study. Lupus 2011;20:1293-9. 